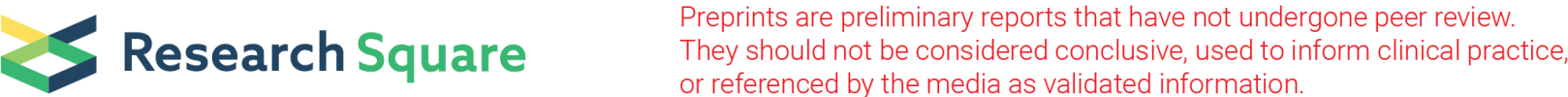

\section{Associations of hysteroscopic features of chronic endometritis with pregnancy outcomes of in vitro fertilization: A retrospective study}

\section{Hui Wang}

Qingdao Women and Children's Hospital, Cheeloo College of Medicine, Shandong University

Chang-hong Liu

Yantaishan Hospital

Cui-fang Hao ( $\square$ wang1980cjy@163.com )

Qingdao Women and Children's Hospital, Cheeloo College of Medicine, Shandong University

\section{Research Article}

Keywords: chronic endometritis, infertility, in vitro fertilization, hysteroscopy

Posted Date: March 5th, 2021

DOI: https://doi.org/10.21203/rs.3.rs-271055/v1

License: (c) (i) This work is licensed under a Creative Commons Attribution 4.0 International License. Read Full License 


\section{Abstract}

The impact of the hysteroscopic features of chronic endometritis (CE) on pregnancy outcomes is unclear. This study explored whether the morphological features of CE on hysteroscopy were associated with in vitro fertilization (IVF) pregnancy outcomes. This retrospective study was conducted at Yantai Yuhuangding Hospital from 01/2017 to 09/2018. Infertile women who underwent hysteroscopy before IVF were grouped according to CE. To decrease confounding, a group of standardized patients was selected from the women enrolled in this study to compare pregnancy outcomes between the $\mathrm{CE}$ and non-CE groups. The outcomes were clinical pregnancy rate (CPR), live birth rate (LBR), miscarriage rate, and premature birth rate. In this study, 3280 women underwent IVF, and 3179 of these patients underwent hysteroscopy. In standardized patients, significant differences were found between the CE and non-CE groups in CPR (54.3\% vs. $65.6 \%, P=0.02)$ and LBR (45.7\% vs. $58.3 \%, P=0.012)$. In patients who underwent fresh embryo transfer, CPR differed among groups $(P=0.002)$ and was highest in the hemorrhagic spots group (61.7\%). In patients who underwent frozen embryo transfer (FET), CPR was higher in the CE group than in the non-CE group (54.7\% vs. $43.0 \%, \mathrm{P}<0.001)$, highest in the hemorrhagic spots group $(70.6 \%, \mathrm{P}=0.002)$ and lowest in the hyperemia combined with micropolyps group $(39.4 \%, \mathrm{P}=0.022)$. The only factor independently associated with CPR was hysteroscopic features of CE (odds ratio: 1.47, 95\% confidence interval: 1.21-1.80, P<0.001). Hysteroscopic features of CE are associated with adverse pregnancy outcomes after IVF.

\section{Introduction}

Assisted reproductive technology (ART) is continuously evolving and can mitigate the impacts of ovulatory dysfunction, diminished ovarian reserve, ovarian failure, tubal disorders, uterine disorders, and male factors ${ }^{1}$. ART allows infertile couples to have children with success rates of up to $65 \%^{1,2}$. Nevertheless, the pregnancy rate has not significantly improved in recent years ${ }^{3}$, and uterine pathologies are still important factors affecting the success of ART ${ }^{4}$.

Chronic endometritis (CE) is a common uterine lesion caused by an imbalance between microorganisms and the host immune system in the endometrium ${ }^{5}$. CE is associated with adverse pregnancy outcomes such as infertility, premature delivery, and miscarriage ${ }^{6,7}$. The prevalence of CE in the general population is approximately $0.8 \%-19 \%$ 8 . In patients with infertility, the prevalence of CE is estimated to be $2.8 \%-39 \%{ }^{9}$ but is even higher $(60 \%-66 \%)$ in women with recurrent pregnancy loss or recurrent implantation failure ${ }^{10}$. CE can reduce the success rate of pregnancy and even lead to obstetric and neonatal complications ${ }^{8,11-14}$. Furthermore, CE is an adverse factor for the success of ART ${ }^{5}$.

CE is usually difficult to diagnose because the clinical signs and symptoms are often attenuated or mild. The triad of endometrial histological examination, hysteroscopy, and microbiology culture might be the best approach for the diagnosis of $\mathrm{CE}{ }^{10}$, but these three methods differ in their specificity, sensitivity, and accuracy for CE diagnosis ${ }^{15}$. Hysteroscopic evaluation of CE might have a higher sensitivity than endometrial microbial culture ${ }^{16}$. Normal endometrial features at hysteroscopy may predict the likelihood of a successful pregnancy ${ }^{17}$. Surprisingly, the histopathologic grade of chronic inflammation does not closely correlate with clinical symptoms ${ }^{18}$. Therefore, considering the relationship between the hysteroscopic features of CE and poor pregnancy outcomes, difficulties in the histopathologic diagnosis of $\mathrm{CE}$, and differences between histopathologic and hysteroscopic results, hysteroscopy might be the best method of evaluating intrauterine inflammation ${ }^{6}$. There are various hysteroscopic 
manifestations of CE, including micropolyps (<1 mm), stromal edema, hemorrhagic spots, and hyperemia, which are either localized or scattered throughout the cavity ${ }^{19-21 .}$

The impact of the different hysteroscopic features of CE on pregnancy outcomes has not been clearly reported. Therefore, the aim of this study was to explore the association between the morphological features of CE at hysteroscopy and pregnancy outcomes after in vitro fertilization (IVF).

\section{Results}

\section{Characteristics of the patients}

The study population comprised 3179 patients underwent 4395 cycles of egg retrieval, with fresh embryo transfer in 1648 cycles, whole embryo freezing in 2191 cycles, and no embryo transfer in 556 cycles. According to the Delphi diagnostic criteria, 767 of the 3179 patients (24.1\%) were diagnosed with CE and 2412 patients (75.9\%) were diagnosed as not having CE. A total of 1035 cycles were performed in the 767 patients with CE, including fresh embryo transfer in 429 cycles, whole embryo freezing in 410 cycles, including 384 FET cycles. and no embryo transfer in 196 cycles. A total of 3360 cycles were performed in the 2412 patients without CE, including fresh embryo transfer in 1219 cycles, whole embryo freezing in 1745 cycles, and no embryo transfer in 396 cycles.

According to the inclusion criteria for the standardized group of patients, a total of 583 standardized cycles were included from the 1648 cycles of fresh embryo transfer performed in the 3179 patients who underwent hysteroscopy. According to the exclusion criteria, 86 standardized cycles were subsequently excluded (recurrent abortions, $n=29$; scarred uterus, $n=19$; complicated by medical diseases, including thyroid disease, hypertension, diabetes or hematologic disease, $n=11$; uterine fibroids, $n=8$; cervical lesions or cervical coning, $n=5$; chromosomal abnormalities in either male or female, $n=5$; hyperprolactinemia, $n=3$; congenital uterine malformation, $n=2$; endometrial cancer, $n=1$; history of intrauterine device use, $n=1$; autoimmune systemic disease, $n=1$; pelvic or genital tuberculosis, $n=1$ ). Therefore, a total of 497 cycles in standardized patients were included in the analysis. There were 436 high-quality embryo transfer cycles and 61 cycles of non-high-quality embryo transfer. Of the 436 high-quality embryo transfer cycles, 116 had CE and 320 had non-CE. Of the 61 non-high-quality embryo transfer cycles, 18 had CE and 43 had non-CE (Supplemental Figure S2). Due to the small number of standardized patients with CE, further stratification for analysis of hysteroscopic manifestations was not performed

Tables 1 and 2 present the clinical characteristics of the patients who underwent fresh embryo transfer and FET, respectively. The patients who underwent fresh embryo transfer were divided into a hemorrhagic spots group $(n=175)$, hyperemia group ( $n=122)$, micropolyps group $(n=75)$, micropolyps combined with hyperemia group $(n=49)$, and other group $(n=8)$ based on the hysteroscopy findings. There were no differences among these groups in age, BMI, type of infertility, and infertility duration (all $\mathrm{P}>0.05$; Table 1 ). The patients who underwent FET were allocated to a CE group $(n=384)$ and non-CE group $(n=1715)$. Furthermore, the patients with CE were divided into a hemorrhagic spots group ( $n=126)$, hyperemia group $(n=112)$, micropolyps group $(n=69)$, micropolyps combined with hyperemia group $(n=66)$, and other group $(n=11)$. There were differences between these groups in age and type of infertility (all $\mathrm{P}<0.05$ ) (Table 2 ).

\section{Pregnancy outcomes}

For the fresh embryo transfer cycles, no significant differences were found between the CE and non-CE groups in CPR (49.0\% vs. 53.1\%, P=0.325), LBR (40.8\% vs. 45.4\%, $P=0.254)$, miscarriage rate (14.8\% vs. $12.2 \%$, $P=0.626)$, or 
premature birth rate (17.1\% vs. 15.5\%, P=0.551) (Figure 2 and Supplementary Table S1). Among the standardized patients who underwent fresh embryo transfer, the CE group had a lower CPR (54.3\% vs. 65.6\%, P=0.020) and LBR ( $45.7 \%$ vs. $58.3 \%, P=0.012$ ) than the non-CE group (Figure 2 and Supplementary Table S2). Among the standardized patients with high-quality embryo transfer, the CE group had a lower CPR $(58.6 \%$ vs. $70.6 \%, P=0.018)$ and LBR $(50.0 \%$ vs. $62.8 \%, \mathrm{P}=0.016)$ and a higher premature birth rate $(25.0 \%$ vs. $11.1 \%, \mathrm{P}=0.004)$ than the non-CE group (Figure 2 and Supplementary Table S3).

\section{Comparison of pregnancy outcomes after IVF between groups based on the hysteroscopic features of CE}

The CPR for the fresh embryo transfer cycles differed among the various types of CE $(P=0.002)$, and the hemorrhagic spots group had the highest CPR (61.7\%, P<0.0083) (Table 3 and Supplementary Table S4). The LBR also differed among the groups $(P=0.011)$ (Table 3$)$ and was highest for the hemorrhagic spots group (51.4\%), with a significant difference between the hemorrhagic spots group and hyperemia group ( $P=0.001)$ (Table 3 and Supplementary Table S4). The miscarriage and premature birth rates were not significantly different between groups $(\mathrm{P}>0.05)$ (Table 3$)$.

Patients with CE underwent anti-inflammatory therapy before FET. The CPR for FET cycles was higher for patients with CE than for patients without CE (54.7\% vs. 43.0\%, P<0.001) (Table 3). The CPR for FET cycles was highest in the hemorrhagic spots group $(70.6 \%, \mathrm{P}=0.002)$ and lowest in the hyperemia combined with micropolyps group (39.4\%, $\mathrm{P}=0.022)$ (Table 4).

\section{Factors associated with CPR and CE}

Multivariable logistic regression analysis showed that hysteroscopic features of CE was the only factor independently associated with CPR in patients with CE (OR=1.47, 95\% Cl: 1.21-1.80, P<0.001) (Table 5). Age, type of infertility, infertility duration, BMI, history of abortion (number of induced abortions and medical abortions), reason for IVF treatment (including polycystic ovarian syndrome [PCOS]), insulin resistance, pelvic inflammatory disease (PID), endometriosis, and male factor infertility were not associated with CPR in patients with CE (all P凶0.05).

The logistic regression analysis also showed that age, induced abortions, PCOS, and hydrosalpinx were independently associated with CE (Supplementary Table S5).

\section{CD138 expression}

Among the 767 patients with CE diagnosed by hysteroscopy, 92 patients underwent endometrial biopsy for determination of CD138 expression (biopsy was refused or not recommended in the remaining patients with $\mathrm{CE}$ ). There were no differences in CD138 expression among between groups stratified according to embryo transfer method (Supplementary Table S6) or type of CE (Supplementary Table S7).

\section{Discussion}

The exact impact of CE on the outcomes of IVF are poorly known. The results strongly suggest that the hysteroscopic features of CE are closely associated with adverse pregnancy outcomes after IVF. Notable, there were differences in pregnancy outcomes between women with different hysteroscopic features of CE.

The present study showed that the frequency of CE in women treated with IVF for infertility was $24.1 \%$. When all the embryo transfer cycles of standardized patients were analyzed, significant differences were found in CPR and 
LBR between women with CE and their non-CE counterparts. The above finding is supported by numerous previous studies ${ }^{10,17,21-23}$. In addition, among the standardized patients who received high-quality embryos, the preterm birth rate was higher for the CE group than for the non-CE group, as observed in a prior study ${ }^{14}$. Therefore, we believe that attention should be paid to the screening and diagnosis of CE in patients undergoing IVF.

Hysteroscopy has gained support as a useful tool for evaluating the intrauterine environment during the IVF process ${ }^{21}$. Because hysteroscopy is a relatively straightforward and low-risk procedure ${ }^{24}$, we suggest that it should be routinely performed in women with infertility, especially those who require IVF therapy. The presence of plasma cells in the endometrial stroma remains the accepted histologic criterion for diagnosing CE ${ }^{25}$, but there is no unified consensus on the minimum number of plasma cells required for $\mathrm{CE}$ diagnosis. Therefore, the clinicohistopathologic diagnosis of CE can vary from center to center ${ }^{26}$. One study reported that the concordance rate of hysteroscopy-diagnosed CE and clinico-histopathologic CE was approximately $57.5 \%-66 \%{ }^{21}$, and another investigation determined that the diagnostic accuracy of hysteroscopy (93.4\%) was higher than that of histopathologic methods ${ }^{6}$. Identifying the various hysteroscopic features of CE makes it possible to refine the general description of CE to specific microscopic manifestations and characteristics ${ }^{27}$. Cicinelli et al. ${ }^{28}$ proposed new diagnostic criteria for hysteroscopic CE (the Delphi consensus) based on international randomized controlled observations. To further explore the diagnostic value of hysteroscopy for $\mathrm{CE}$, we compared the IVF pregnancy outcomes between patients with different hysteroscopic features of CE. The CPR and LPR were low in patients with CE that manifested as hyperemia, micropolyps, or both under hysteroscopy. Therefore, careful attention should be paid to identifying and treating these microscopic manifestations of CE because they are associated with poor pregnancy outcomes. Studies have shown that the prophylactic use of antibiotics can improve pregnancy outcomes in patients with recurrent implantation failure and recurrent pregnancy loss ${ }^{10,21,29}$. Interestingly, the pregnancy outcomes were significantly better in the hemorrhagic spots group than in the other groups, and this trend was seen consistently both for fresh embryo transfer cycles and FET cycles. We suspect that hemorrhagic spots may represent a condition other than overt CE, but since only a small number of patients underwent biopsy and histopathologic examination, this will have to be examined further in future studies. A prior investigation determined that focal or scattered red areas observed by hysteroscopy occur when circulating estrogen levels are high ${ }^{30}$. The influence of hemorrhagic spots on the intrauterine environment needs further research. Therefore, at present, we suggest that anti-inflammatory treatment may not be necessary for patients with CE who present with hemorrhagic spots at hysteroscopy.

A previous randomized controlled trial ${ }^{23}$ concluded that histopathologic CE did not affect IVF outcomes.

Furthermore, another study ${ }^{31}$ suggested that histopathologic CE was associated with the incidence of reproductive disorders in patients with clinical symptoms of PID. The above results imply that a histopathologic diagnosis of CE does not indicate which patients would benefit from further treatment to improve pregnancy outcomes ${ }^{5}$. On the other hand, the present study showed that the hysteroscopic features of CE were independently associated with CPR. In future, it is possible that microbiome studies or hysteroscopy may replace histopathology as the goldstandard tool for diagnosing $\mathrm{CE}^{32}$.

Our analysis showed that endometriosis was not significantly associated with the occurrence of CE, in contrast to the results of a previous study ${ }^{13}$. Indeed, endometriosis is the ectopic growth of endometrial tissue outside the uterine cavity, whereas CE is a pathologic process involving an imbalance in the microbiota. Nevertheless, CE has been shown to contribute to the transformation of normal endometrial tissue into invasive endometrial tissue that 
can invade the pelvic cavity ${ }^{33}$. The lack of an association between CE and endometriosis in this study might be due to a short CE course or the use of antibiotics. This will have to be examined in future studies.

At present, many authors believe that pathologic examination is the gold standard diagnostic method for CE. However, in recent years, some studies have pointed out that a pathologic diagnosis does not adequately reflect the inflammatory state 10,17,18,20,24,28, which could introduce a bias. Moreover, the histologic alterations of CE may be focal, normally present in the endometrial mucosa, and not homogenously distributed. Therefore, it is possible that a pathologist might underestimate the extent of CE. Supporting this concept, although only 92 patients received a pathologic examination, there was no association between CD138 positivity and pregnancy outcomes. On the other hand, the hysteroscopic view allows a thorough evaluation of the entire uterine cavity. In view of the limitations of histopathology for the diagnosis of $\mathrm{CE}$, inconsistent results between histopathologic and hysteroscopic diagnoses, and the association between hysteroscopic features of CE and poor pregnancy outcomes ${ }^{6}$ hysteroscopy-guided biopsy may be a superior strategy to curettage in the diagnosis of CE. When endometrial tissue is required for pathologic examination, we would encourage the collection of samples under hysteroscopic guidance rather than by blind curettage. In other words, hysteroscopy should be performed first, with tissue samples collected during hysteroscopy if any abnormalities are observed. However, further randomized controlled trials are needed to establish a unified set of criteria for the diagnosis of CE by hysteroscopy. Additionally, since the identification of CE at hysteroscopy involves the judgement of the operator, it is possible that the diagnosis might be missed if the procedure is performed by an inexperienced clinician. Therefore, research will be needed to investigate inter-observer variation and learning curves for CE diagnosis.

This study has some limitations. The hysteroscopic diagnosis of CE relied on subjective features, and the examinations were performed by different doctors. Nevertheless, the influence of different operators is considered to be minimal ${ }^{34,35}$. Furthermore, the diagnostic criteria for CE at hysteroscopy used in this study were not identical

to those described in the recently published Delphi consensus ${ }^{28}$. Nevertheless, our criteria were similar to those of the Delphi consensus and were based on previously reported features of CE ${ }^{19-21}$. In addition, only 92 patients in this study received a histopathologic diagnosis. Among these 92 patients, the endometrial biopsies were obtained blindly by curettage rather than under hysteroscopic guidance, so it is possible that CE may have been underdiagnosed. Nevertheless, this was the routine practice at this hospital during the study period. The present analysis also has limitations inherent to retrospective observational studies. Therefore, randomized controlled trials are needed to confirm our findings.

In conclusion, the present study indicates that the hysteroscopic features of CE are associated with adverse pregnancy outcomes after IVF, since there were differences in pregnancy outcomes between women with different hysteroscopic features of CE. These findings suggest that the diagnosis of CE by hysteroscopy may have potential for broader application. A consensus on the relationship between diagnostic criteria and pregnancy outcomes is essential for the provision of effective and individualized treatment for infertility. We hope that the findings of this study will help emphasize the importance of this goal.

\section{Materials And Methods}

\section{Study design and patients}

This retrospective study was conducted at the Center for Reproductive Medicine of Yantai Yuhuangding Hospital and included women admitted from January 2017 to September 2018. The inclusion criteria were: 1) infertility; 2) 
underwent IVF treatment; and 3) hysteroscopy was performed within 3 months prior to IVF in order to diagnose CE according to Delphi's diagnostic criteria ${ }^{28}$. The exclusion criteria were: 1 ) dilated endometrial vessels; 2 ) refused hysteroscopy; or 3) not suitable for hysteroscopy (suspected acute reproductive infection, menstruation at the time of examination, unexplained uterine bleeding, or a positive result on a pregnancy test).

In order to exclude possible confounding factors that might influence the pregnancy outcomes, such as age, cause of infertility, combined disease, follicular stimulation protocol, and quality of the transplanted embryos, a group of standardized patients was selected from the participants enrolled in this study according to the following standards: 1) <35 years of age; 2 ) normal ovarian reserve function (antral follicle count $>7$, anti-Müllerian hormone level of $1.0-4.0 \mathrm{ng} / \mathrm{mL}$, and baseline follicle stimulating hormone [FSH] level $<10 \mathrm{IU} / \mathrm{L}$ ) ${ }^{36} ; 3$ ) standard long-term protocol for follicle stimulation; 4) fallopian tube obstruction as the single infertility factor for IVF treatment; and 5) underwent fresh embryo transfer. The exclusion criteria were: 1) endometrial carcinoma; 2) comorbidities including thyroid disease, hypertension, diabetes, and hematologic diseases; 3) hyperprolactinemia; 4) cervical lesions or conization of the cervix; 5) intrauterine devices; 6) uterine fibroids; 7) autoimmune diseases; 8) chromosomal abnormalities in either male or female family members; 9) recurrent abortions; 10) congenital uterine malformations; 11) pelvic or genital tuberculosis; and 12) uterine scarring.

This study was approved by the ethics committee of Yantai Yuhuangding Hospital. All data were extracted from the database of the Center for Reproductive Medicine of Yantai Yuhuangding Hospital. All methods were performed in accordance with the relevant guidelines. The patient's informed consent was obtained for inclusion in the database.

\section{Grouping}

The included patients were divided into the CE group (diagnosed with CE) and non-CE group (not diagnosed with $\mathrm{CE}$ ) based on the hysteroscopy findings. Then, the patients with CE were divided into five subgroups according to the hysteroscopic characteristics: hemorrhagic spots, hyperemia (including diffuse hyperemia and focal hyperemia), micropolyps, hyperemia combined with micropolyps, and other.

The diagnostic criteria for CE at hysteroscopy were based on Delphi's diagnostic criteria ${ }^{28:}$ 1) diffuse hyperemia: large areas of hyperemia with white points (Figure 1A);2) focal hyperemia: small areas of hyperemia (Figure 1B); 3) hemorrhagic spots: focal red areas with sharp and irregular borders possibly in continuity with a capillary (Figure 1C); 4) micropolyps: endometrial polyps $<1 \mathrm{~mm}$ in diameter with prominent vascular pedicles, distributed focally (Figure 1D) or diffusely (Figure 1E); and 5) stromal edema: thick and pale appearance of the follicular endometrium (a normal finding during the secretory phase, Figure 1F). The diagnosis and classification of CE were performed by two physicians who had received professional training.

The histopathologic diagnostic criterion for $\mathrm{CE}^{6}$ was the detection of plasma cell-specific surface antigen CD138 by immunohistochemistry. CE was diagnosed if at least five plasma cells were counted in the endometrial stroma in each randomly chosen high-magnification field (×400) using an Olympus (Tokyo, Japan) microscope.

\section{Management of CE}

The patients with CE scheduled for frozen embryo transfer (FET) received treatment for CE: oral doxycycline 100 mg bid plus metronidazole tablets $0.4 \mathrm{~g}$ tid for 2 weeks, or cefdinir dispersible tablets $100 \mathrm{mg}$ tid orally plus oral metronidazole tablets $0.4 \mathrm{~g}$ tid for 2 weeks. At the same time, some patients used traditional Chinese medicine 
enema for 10 days after the end of menstruation and for two consecutive menstrual cycles. The main treatment to improve the endometrial receptivity of patients with CE who were scheduled for FET was down-regulation and induction of an artificial cycle. The patients with CE who were scheduled for fresh embryo transfer were advised to undergo whole embryo freezing and then to undergo frozen embryo transplantation after anti-inflammatory therapy for CE mentioned above. For the patients with CE who were scheduled for fresh embryo transfer but refused anti-inflammatory therapy, or the patients with CE who had mild inflammation that did not qualify for antiinflammatory treatment mentioned above, prophylactic antibiotic treatment (second-generation oral cephalosporin tid for 3 days) after hysteroscopy was used.

\section{IVF protocol}

Before IVF treatment, all patients with CE were fully informed regarding the inflammatory status of their uterine cavity. With the informed consent of the patient, whole embryo freezing was performed after oocyte retrieval to allow for anti-inflammatory treatment for CE to be administered. If anti-inflammatory treatment was refused or not recommended due to mild inflammation, embryo transfer in the cleavage stage was performed on the third day after oocyte retrieval. The gonadotropin-releasing hormone $(\mathrm{GnRH})$ agonist regimen was used as the standard long-term protocol and involved the daily injection of $0.05 \mathrm{mg}$ triptorelin acetate (Ipsen; Boulogne-Billancourt) in the mid-luteal period of the preceding menstrual cycle. Pituitary suppression (luteinizing hormone level $<5 \mathrm{IU} / \mathrm{L}$, estradiol level $<50 \mathrm{ng} / \mathrm{L}$, endometrial thickness $<5 \mathrm{~mm}$, and no functional ovarian cyst) was achieved after 14 days. The dose of recombinant FSH (Gonal F; Serono, Rockland, MA) or purified urinary human menopausal gonadotropin (Repronex; Ferring Pharmaceuticals, Suffern, NY) was adjusted (75-300 U/d) to achieve ovarian stimulation. When at least one follicle was $>17 \mathrm{~mm}$ in diameter, 4000-10000 IU of human chorionic gonadotropin (hCG) was administered subcutaneously, and ultrasound-guided transvaginal oocyte retrieval was performed 35 hours later. Embryo transfer in the cleavage stage was performed on the third day after oocyte retrieval. All patients undergoing fresh embryo transfer received luteal support until pregnancy. The dosing was stopped after 10 weeks. Serum hCG level was measured 14 days after embryo transfer, and ultrasound was performed 28 days after embryo transfer.

\section{Hysteroscopy and endometrial biopsy}

Since there is some evidence that hysteroscopy can improve outcomes after ART (Di Spiezio Sardo et al., 2016), our center recommends that all patients undergo hysteroscopy before IVF. Hysteroscopy was scheduled for day 612 of the menstrual cycle. The procedure was performed using a rigid hysteroscope with a 3.5-mm-diameter outer sheath and $30^{\circ}$ viewing angle (Karl Storz, Germany). Saline $(0.9 \%)$ was used as the medium at $100 \mathrm{mmHg}$ pressure. All hysteroscopies were performed by two physicians who had received professional training. The video results were recorded in the MEDCON medical information technology network system. For patients who consented, the endometrium was sampled blindly at the end of hysteroscopy using a metal curette for endometrial biopsy. All patients received prophylactic oral antibiotic therapy (Cefuroxime ester tablets 250mg bid) for 2 days after hysteroscopy.

\section{Data collection}

The clinical data of all patients were retrieved from the Wuhan Mutual Creation Assisted Reproductive Information Management System, including age, infertility duration, type of infertility, cause of infertility, initial diagnosis, body mass index (BMI), ovarian reserve function, mode of ART, indications, medication protocol, oocyte retrieval, embryo transfer, frozen embryo condition, FET, clinical pregnancy rate (CPR), live birth rate (LBR), premature birth rate, and 
miscarriage rate. Previous medical records, including past medical history, obstetric history, fallopian tube examination findings, and endometrial histology results, were also retrieved from Wuhan Mutual Creation Assisted Reproductive Information Management System. The primary outcome of the study was CPR, and secondary outcomes were LBR, premature birth rate, and miscarriage rate.

\section{Definitions}

A high-quality embryo was defined as a grade 1-2 embryo comprising 7-9 cells ${ }^{37}$. Blastocyst quality was evaluated according to the Gardner scoring system ${ }^{7}$, and high-quality blastocysts were defined as having embryo scores greater than 3BB, which excluded the inner cell mass and trophoblastic layer C. Clinical pregnancy was defined as one or more pregnancy sacs identified during ultrasonography. Biochemical pregnancies were not included ${ }^{36}$. The CPR was defined as the number of clinical pregnancy cycles/number of embryo transfer cycles $\times$ $100 \%{ }^{36}$. The LBR was defined as the number of live births/number of embryo transfer cycles $\times 100 \%{ }^{36}$. The miscarriage rate was defined as the number of cycles with spontaneous abortion within 28 weeks/number of clinical pregnancy cycles $\times 100 \%{ }^{36}$. The premature birth rate was defined as the number of birth cycles within $28-$ 37 weeks/number of clinical pregnancy cycles $\times 100 \%{ }^{36}$.

\section{Follow-up}

All patients were followed-up by nursing staff. Blood hCG level was measured 14 days after transplantation. A vaginal ultrasound examination was performed 28 days after transplantation. Ultrasound was repeated at 10 weeks of pregnancy. Follow-ups were conducted during mid-pregnancy, late pregnancy, and after childbirth by telephone to record any comorbidities.

\section{Statistical analysis}

All data were analyzed using SPSS 21.0 for Windows (IBM, Armonk, NY, USA). Continuous data were tested for a normal distribution using the Kolmogorov-Smirnov test. Normally-distributed continuous data are presented as means \pm standard deviations and were analyzed using the t-test for independent samples or single-sample ANOVA. Non-normally-distributed continuous data are presented as medians (ranges) and were analyzed using the Wilcoxon rank-sum test. Categorical data were analyzed using the chi-squared test, Fisher's exact test, or the corrected chi-squared test, as appropriate. Factors associated with pregnancy outcomes were identified using univariable and multivariable logistic regression analysis with stepwise selection, and odds ratios (ORs) and 95\% confidence intervals $(95 \% \mathrm{Cls})$ were calculated. $\mathrm{P}<0.05$ was taken to indicate statistical significance.

\section{Declarations}

\section{Acknowledgments}

The authors acknowledge the Center for Reproductive Medicine in Yantai Yuhuangding Hospital for the teamwork that generated this manuscript. We thank all the patients who participated in our research.

\section{Declaration of interest}

None.

\section{Funding}


No funding.

\section{References}

1. Lindsay, T. J. \& Vitrikas, K. R. Evaluation and treatment of infertility. Am Fam Physician. 91, 308-314 (2015).

2. McLernon, D. J., Steyerberg, E. W., Te Velde, E. R., Lee, A. J. \& Bhattacharya, S. Predicting the chances of a live birth after one or more complete cycles of in vitro fertilisation: population based study of linked cycle data from 113873 women. BMJ. 355, i5735 (2016).

3. Adegboyega, P. A., Pei, Y. \& McLarty, J. Relationship between eosinophils and chronic endometritis. Hum Pathol. 41, 33-37 (2010).

4. Galliano, D., Bellver, J., Diaz-Garcia, C., Simon, C. \& Pellicer, A. ART and uterine pathology: how relevant is the maternal side for implantation? Hum Reprod Update. 21, 13-38 (2015).

5. Park, H. J., Kim, Y. S., Yoon, T. K. \& Lee, W. S. Chronic endometritis and infertility. Clin Exp Reprod Med. 43, 185192 (2016).

6. Bouet, P. E., et al. Chronic endometritis in women with recurrent pregnancy loss and recurrent implantation failure: prevalence and role of office hysteroscopy and immunohistochemistry in diagnosis. Fertil Steril. 105, 106-110 (2016).

7. Smith, M., Hagerty, K. A., Skipper, B. \& Bocklage, T. Chronic endometritis: a combined histopathologic and clinical review of cases from 2002 to 2007. Int J Gynecol Pathol. 29, 44-50 (2010).

8. Feghali, J., et al. [Systematic hysteroscopy prior to in vitro fertilization]. Gynecol Obstet Fertil. 31, 127-131 (2003).

9. Kasius, J. C., et al. The reliability of the histological diagnosis of endometritis in asymptomatic IVF cases: a multicenter observer study. Hum Reprod. 27, 153-158 (2012).

10. Cicinelli, E., et al. Prevalence of chronic endometritis in repeated unexplained implantation failure and the IVF success rate after antibiotic therapy. Hum Reprod. 30, 323-330 (2015).

11. Espinoza, J., Erez, O. \& Romero, R. Preconceptional antibiotic treatment to prevent preterm birth in women with a previous preterm delivery. Am J Obstet Gynecol. 194, 630-637 (2006).

12. Gravett, M. G., Hitti, J., Hess, D. L. \& Eschenbach, D. A. Intrauterine infection and preterm delivery: evidence for activation of the fetal hypothalamic-pituitary-adrenal axis. Am J Obstet Gynecol. 182, 1404-1413 (2000).

13. Kitaya, K., et al. Chronic Endometritis: Potential Cause of Infertility and Obstetric and Neonatal Complications. Am J Reprod Immunol. 75, 13-22 (2016).

14. Knudtson, E. J., et al. The association of chronic endometritis with preterm birth. Am J Obstet Gynecol. 196, 337 e331-334 (2007).

15. Song, D., et al. Prevalence and confounders of chronic endometritis in premenopausal women with abnormal bleeding or reproductive failure. Reprod Biomed Online. 36, 78-83 (2018).

16. Bakas, P., et al. Role of hysteroscopy prior to assisted reproduction techniques. J Minim Invasive Gynecol. 21, 233-237 (2014).

17. Cicinelli, E., et al. Chronic endometritis due to common bacteria is prevalent in women with recurrent miscarriage as confirmed by improved pregnancy outcome after antibiotic treatment. Reprod Sci. 21, 640-647 (2014). 
18. Levi Setti, P. E., et al. Implantation failure in assisted reproduction technology and a critical approach to treatment. Ann N Y Acad Sci. 1034, 184-199 (2004).

19. Chen, Y., et al. Prevalence and Impact of Chronic Endometritis in Patients With Intrauterine Adhesions: A Prospective Cohort Study. J Minim Invasive Gynecol. 24, 74-79 (2017).

20. Cicinelli, E., et al. Endometrial micropolyps at fluid hysteroscopy suggest the existence of chronic endometritis. Hum Reprod. 20, 1386-1389 (2005).

21. Yang, R., et al. The hysteroscopy and histological diagnosis and treatment value of chronic endometritis in recurrent implantation failure patients. Arch Gynecol Obstet. 289, 1363-1369 (2014).

22. Andrews, W. W., et al. Endometrial microbial colonization and plasma cell endometritis after spontaneous or indicated preterm versus term delivery. Am J Obstet Gynecol. 193, 739-745 (2005).

23. Kasius, J. C., et al. The impact of chronic endometritis on reproductive outcome. Fertil Steril. 96, 1451-1456 (2011).

24. Cicinelli, E. Diagnostic minihysteroscopy with vaginoscopic approach: rationale and advantages. J Minim Invasive Gynecol. 12, 396-400 (2005).

25. Chen, Y. Q., Fang, R. L., Luo, Y. N. \& Luo, C. Q. Analysis of the diagnostic value of CD138 for chronic endometritis, the risk factors for the pathogenesis of chronic endometritis and the effect of chronic endometritis on pregnancy: a cohort study. BMC Womens Health. 16, 60 (2016).

26. Groth, J. V. Chronic endometritis and the plasma cell, fact versus fiction. Fertil Steril. 109, 788 (2018).

27. Radzinsky, V. E., Kostin, I. N., Petrov, Y. A., Polina, M. L. \& Gasanova, B. M. Diagnostic significance of chronic endometritis macrotypes differentiation among women with reproductive losses. Gynecol Endocrinol. 33, 3640 (2017).

28. Cicinelli, E., et al. Unified diagnostic criteria for chronic endometritis at fluid hysteroscopy: proposal and reliability evaluation through an international randomized-controlled observer study. Fertil Steril. 112, 162-173 e162 (2019).

29. McQueen, D. B., Bernardi, L. A. \& Stephenson, M. D. Chronic endometritis in women with recurrent early pregnancy loss and/or fetal demise. Fertil Steril. 101, 1026-1030 (2014).

30. Chianeh, Y. R. \& Rao, P. Molecular and hormonal regulation of angiogenesis in proliferative endometrium. Int $J$ Res Med Sci. 2, 1-9 (2014).

31. Haggerty, C. L., et al. Endometritis does not predict reproductive morbidity after pelvic inflammatory disease. Am J Obstet Gynecol. 188, 141-148 (2003).

32. Moreno, I., et al. The diagnosis of chronic endometritis in infertile asymptomatic women: a comparative study of histology, microbial cultures, hysteroscopy, and molecular microbiology. Am J Obstet Gynecol. 218, 602 e601-602 e616 (2018).

33. Takebayashi, A., et al. The association between endometriosis and chronic endometritis. PLoS One. 9, e88354 (2014).

34. Kitaya, K., et al. Local mononuclear cell infiltrates in infertile patients with endometrial macropolyps versus micropolyps. Hum Reprod. 27, 3474-3480 (2012).

35. Song, D., et al. Correlation between hysteroscopy findings and chronic endometritis. Fertil Steril. 111, 772-779 (2019).

36. Hu, L. L., Huang, G. N. \& Sun, H. X. CSRM guidelines consensus specification (2016). Reprod Med. 26, 289-301 (2017). 
37. Jiang, L., et al. A Chinese practice guideline of the assisted reproductive technology strategies for women with advanced age. J Evid Based Med. 12, 167-184 (2019).

\section{Tables}

Table 1. Clinical characteristics and hysteroscopic features of chronic endometritis (CE) in patients with CE who underwent fresh embryo transfer.

\begin{tabular}{|c|c|c|c|c|c|c|}
\hline \multirow[t]{2}{*}{ Characteristic } & \multicolumn{6}{|c|}{ Patients with CE underwent fresh embryo transfer $(n=429)$} \\
\hline & $\begin{array}{l}\text { Hemorrhagic } \\
\text { spots } \\
(n=175 \\
40.8 \%)\end{array}$ & $\begin{array}{l}\text { Hyperemia } \\
(n=122, \\
28.4 \%)\end{array}$ & $\begin{array}{l}\text { Micropolyps } \\
(n=75 \\
17.5 \%)\end{array}$ & $\begin{array}{l}\text { Micropolyps } \\
\text { combined with } \\
\text { hyperemia } \\
(n=49,11.4 \%)\end{array}$ & $\begin{array}{l}\text { Other } \\
(n=8 \\
1.9 \%)\end{array}$ & $\mathrm{P}$ \\
\hline Age, years & $31(29-34)$ & $\begin{array}{l}31(28- \\
34)\end{array}$ & $32(30-36)$ & $31(29-34.5)$ & $\begin{array}{l}32.5(30- \\
35)\end{array}$ & 0.197 \\
\hline $\begin{array}{l}\text { Body mass } \\
\text { index, } \mathrm{kg} / \mathrm{m}^{2}\end{array}$ & $\begin{array}{l}23.7(21.5- \\
26.7)\end{array}$ & $\begin{array}{l}23.4 \\
(20.9- \\
27.2)\end{array}$ & $\begin{array}{l}24.2(22- \\
26.8)\end{array}$ & $22.8(21-27.2)$ & $\begin{array}{l}23.6 \\
(22.7- \\
26.1)\end{array}$ & 0.850 \\
\hline $\begin{array}{l}\text { Types of } \\
\text { infertility, n (\%) }\end{array}$ & & & & & & 0.310 \\
\hline $\begin{array}{l}\text { Primary } \\
\text { infertility }\end{array}$ & $90(51.43 \%)$ & $\begin{array}{l}65 \\
(53.28 \%)\end{array}$ & $31(41.33 \%)$ & $29(59.18 \%)$ & $5(62.5 \%)$ & \\
\hline $\begin{array}{l}\text { Secondary } \\
\text { infertility }\end{array}$ & $85(48.57 \%)$ & $\begin{array}{l}57 \\
(46.72 \%)\end{array}$ & $44(58.67 \%)$ & $20(40.81 \%)$ & $3(37.5 \%)$ & \\
\hline $\begin{array}{l}\text { Infertility } \\
\text { duration, years }\end{array}$ & $3.5(2-5.5)$ & $3.25(2-5)$ & $3.5(2-6.5)$ & $4(2-5)$ & $\begin{array}{l}5.5(4.3- \\
6)\end{array}$ & 0.490 \\
\hline
\end{tabular}

Table 2. Clinical characteristics and hysteroscopic features of chronic endometritis (CE) in patients with CE who underwent frozen embryo transfer. 


\begin{tabular}{|c|c|c|c|c|c|c|c|}
\hline \multirow[t]{2}{*}{ Characteristic } & \multicolumn{6}{|c|}{$\begin{array}{l}\text { Patients with CE underwent frozen embryo transfer } \\
(n=384,18.3 \%)\end{array}$} & \multirow{2}{*}{$\begin{array}{l}\begin{array}{l}\text { Non-CE } \\
\text { group } \\
(n=1715 \text {, } \\
81.7 \%)\end{array}\end{array}$} \\
\hline & $\begin{array}{l}\text { Hemorrhagic } \\
\text { Spots } \\
(n=126)\end{array}$ & $\begin{array}{l}\text { Hyperemia } \\
(\mathrm{n}=112)\end{array}$ & $\begin{array}{l}\text { Micropolyps } \\
(\mathrm{n}=69)\end{array}$ & $\begin{array}{l}\text { Micropolyps } \\
\text { combined } \\
\text { with } \\
\text { hyperemia } \\
(\mathrm{n}=66)\end{array}$ & $\begin{array}{l}\text { Other } \\
(n=11)\end{array}$ & $p$ & \\
\hline Age, years & $31(29-35)$ & $\begin{array}{l}32(29- \\
36)\end{array}$ & $34(30-40)$ & $32(30-35)$ & $\begin{array}{l}34(31- \\
36)\end{array}$ & $<0.001$ & $\begin{array}{l}34(30- \\
38)\end{array}$ \\
\hline $\begin{array}{l}\text { Body mass } \\
\text { index, } \mathrm{kg} / \mathrm{m}^{2}\end{array}$ & $\begin{array}{l}23.9(22- \\
26)\end{array}$ & $\begin{array}{l}24.2(22- \\
26.9)\end{array}$ & $\begin{array}{l}23.4(21.4- \\
25)\end{array}$ & $\begin{array}{l}22.8(20.9- \\
25)\end{array}$ & $\begin{array}{l}22.4 \\
(19.1- \\
27)\end{array}$ & 0.030 & $\begin{array}{l}23.2 \\
(21-26)\end{array}$ \\
\hline $\begin{array}{l}\text { Types of } \\
\text { infertility, n } \\
(\%)\end{array}$ & & & & & & 0.001 & \\
\hline $\begin{array}{r}\text { Primary } \\
\text { infertility }\end{array}$ & 70 (55.56\%) & $\begin{array}{l}60 \\
(53.57 \%)\end{array}$ & $28(40.58 \%)$ & $23(34.85 \%)$ & $\begin{array}{l}6 \\
(54.55 \%)\end{array}$ & & $\begin{array}{l}780 \\
(45.48 \%)\end{array}$ \\
\hline $\begin{array}{l}\text { Secondary } \\
\text { infertility }\end{array}$ & $56(44.44 \%)$ & $\begin{array}{l}52 \\
(46.43 \%)\end{array}$ & 41 (59.42\%) & $43(65.15 \%)$ & $\begin{array}{l}5 \\
(45.45 \%)\end{array}$ & & $\begin{array}{l}935 \\
(54.52 \%)\end{array}$ \\
\hline $\begin{array}{l}\text { Infertility } \\
\text { duration, } \\
\text { years }\end{array}$ & $3.5(2-6)$ & $\begin{array}{l}3.8(2- \\
5.4)\end{array}$ & $3(2-7.3)$ & $3.75(3-5)$ & $4(3-7)$ & 0.210 & $3(2-5)$ \\
\hline $\begin{array}{l}\text { Frozen } \\
\text { embryo cycle } \\
\text { type, } \mathrm{n}(\%)\end{array}$ & & & & & & 0.290 & \\
\hline $\begin{array}{l}\text { Natural } \\
\text { cycle }\end{array}$ & $36(28.57 \%)$ & $\begin{array}{l}27 \\
(24.11 \%)\end{array}$ & $17(24.64 \%)$ & $21(31.82 \%)$ & $\begin{array}{l}4 \\
(36.36 \%)\end{array}$ & & $\begin{array}{l}537 \\
(31.31 \%)\end{array}$ \\
\hline $\begin{array}{l}\text { Artificial } \\
\text { cycle }\end{array}$ & $60(47.62 \%)$ & $\begin{array}{l}44 \\
(39.29 \%)\end{array}$ & 25 (36.23\%) & $33(50.00 \%)$ & $\begin{array}{l}4 \\
(36.36 \%)\end{array}$ & & $\begin{array}{l}674 \\
(39.30 \%)\end{array}$ \\
\hline $\begin{array}{l}\text { Ovulatory } \\
\text { cycle }\end{array}$ & 0 & $2(1.79 \%)$ & $4(5.80 \%)$ & 0 & 0 & & $\begin{array}{l}28 \\
(1.63 \%)\end{array}$ \\
\hline $\begin{array}{l}\text { Post- } \\
\text { reduction } \\
\text { artificial cycle }\end{array}$ & $30(23.81 \%)$ & $\begin{array}{l}39 \\
(34.82 \%)\end{array}$ & $23(33.33 \%)$ & $12(18.18 \%)$ & $\begin{array}{l}3 \\
(27.27 \%)\end{array}$ & & $\begin{array}{l}476 \\
(27.76)\end{array}$ \\
\hline
\end{tabular}

Table 3. IVF pregnancy outcomes according to the hysteroscopic features of chronic endometritis (CE) in patients who underwent fresh embryo transfer cycles. 


\begin{tabular}{|lllll|}
\hline Hysteroscopic feature & CPR $\%$ & LBR $\%$ & $\begin{array}{l}\text { Miscarriage rate } \\
\%\end{array}$ & $\begin{array}{l}\text { Premature birth rate } \\
\%\end{array}$ \\
\hline Hemorrhagic spots $(\mathrm{n}=175)$ & 61.7 & 51.4 & 15.7 & 14.8 \\
\hline Hyperemia $(\mathrm{n}=122)$ & 41.8 & 31.2 & 19.6 & 19.6 \\
\hline Micropolyps $(\mathrm{n}=75)$ & 40.0 & 37.3 & 6.7 & 20.0 \\
$\begin{array}{l}\text { Micropolyps combined with hyperemia } \\
(\mathrm{n}=49)\end{array}$ & 36.7 & 34.7 & 5.6 & 22.2 \\
\hline $\begin{array}{l}\text { Other }(\mathrm{n}=8) \\
P\end{array}$ & 37.5 & 25.0 & 12.5 & 0 \\
\hline P & $0.002^{\star}$ & $0.011 *$ & 0.568 & 0.891 \\
\hline
\end{tabular}

CPR: clinical pregnancy rate; LBR: live birth rate.

$\star P<0.05$.

Table 4. IVF pregnancy outcomes according to the hysteroscopic features of chronic endometritis (CE) in patients who underwent frozen embryo transfer (FET) cycles.

\begin{tabular}{|llll|}
\hline Group & $\mathrm{n}$ & CPR\% & $\mathrm{P}$ \\
\hline FET cycles ${ }^{*}$ in CE group & 384 & 54.7 & \\
\hline FET cycles\# in non-CE group & 1715 & 43.0 & $<0.001$ \\
\hline Hysteroscopic features & & & \\
\hline Hemorrhagic spots & 126 & 70.6 & $0.002^{*}$ \\
\hline Hyperemia & 112 & 52.7 & 0.707 \\
\hline Micropolyps & 69 & 44.9 & 0.135 \\
\hline Micropolyps combined with hyperemia & 66 & 39.4 & $0.022^{*}$ \\
\hline Other & 11 & 45.5 & 0.544 \\
\hline
\end{tabular}

\# First frozen embryo transfer among the entire embryo freezing cycle.

CE: chronic endometritis; CPR: clinical pregnancy rate.

$* P<0.05$.

Table 5. Association between hysteroscopic features of chronic endometritis (CE) and clinical pregnancy rate (CPR) in patients with CE. 


\begin{tabular}{|lllll|}
\hline Variables & Univariable analysis & \multicolumn{2}{l}{ Multivariable analysis } \\
\cline { 2 - 5 } & Odds ratio & $P$ & Odds ratio & P \\
& $(95 \% \mathrm{Cl})$ & & $(95 \% \mathrm{Cl})$ & \\
\hline Age, years & $0.97(0.92-1.01)$ & 0.157 & $0.97(0.91-1.02)$ & 0.224 \\
\hline Body mass index, $\mathrm{kg} / \mathrm{m}^{2}$ & $0.96(0.92-0.99)$ & 0.020 & $0.95(0.90-1.00)$ & 0.066 \\
\hline Type of infertility, $\mathrm{n}(\%)$ & $0.97(0.66-1.42)$ & 0.877 & $1.05(0.62-1.80)$ & 0.85 \\
\hline Infertility duration, years & $1.01(0.94-1.09)$ & 0.786 & $1.04(0.95-1.14)$ & 0.353 \\
\hline Number of miscarriages & $0.97(0.73-1.29)$ & 0.826 & $0.99(0.67-1.46)$ & 0.953 \\
\hline Reason for IVF treatment, $\mathrm{n}(\%)$ & & & & \\
\hline Polycystic ovarian syndrome & $0.74(0.45-1.20)$ & 0.228 & $0.82(0.47-1.42)$ & 0.475 \\
\hline Insulin resistance & $0.66(0.38-0.95)$ & 0.031 & $0.80(0.48-1.36)$ & 0.413 \\
\hline Endometriosis & $1.91(0.98-3.71)$ & 0.058 & $1.69(0.83-3.47)$ & 0.150 \\
\hline Male factor infertility & $0.74(0.41-1.31)$ & 0.302 & $0.69(0.38-1.28)$ & 0.241 \\
\hline Hysteroscopic features of CE & $1.46(1.20-1.77)$ & $<0.001$ & $1.47(1.21-1.8)$ & $<0.001$ \\
\hline
\end{tabular}

Cl: confidence interval.

${ }^{*} \mathrm{P}<0.05$.

Figures 


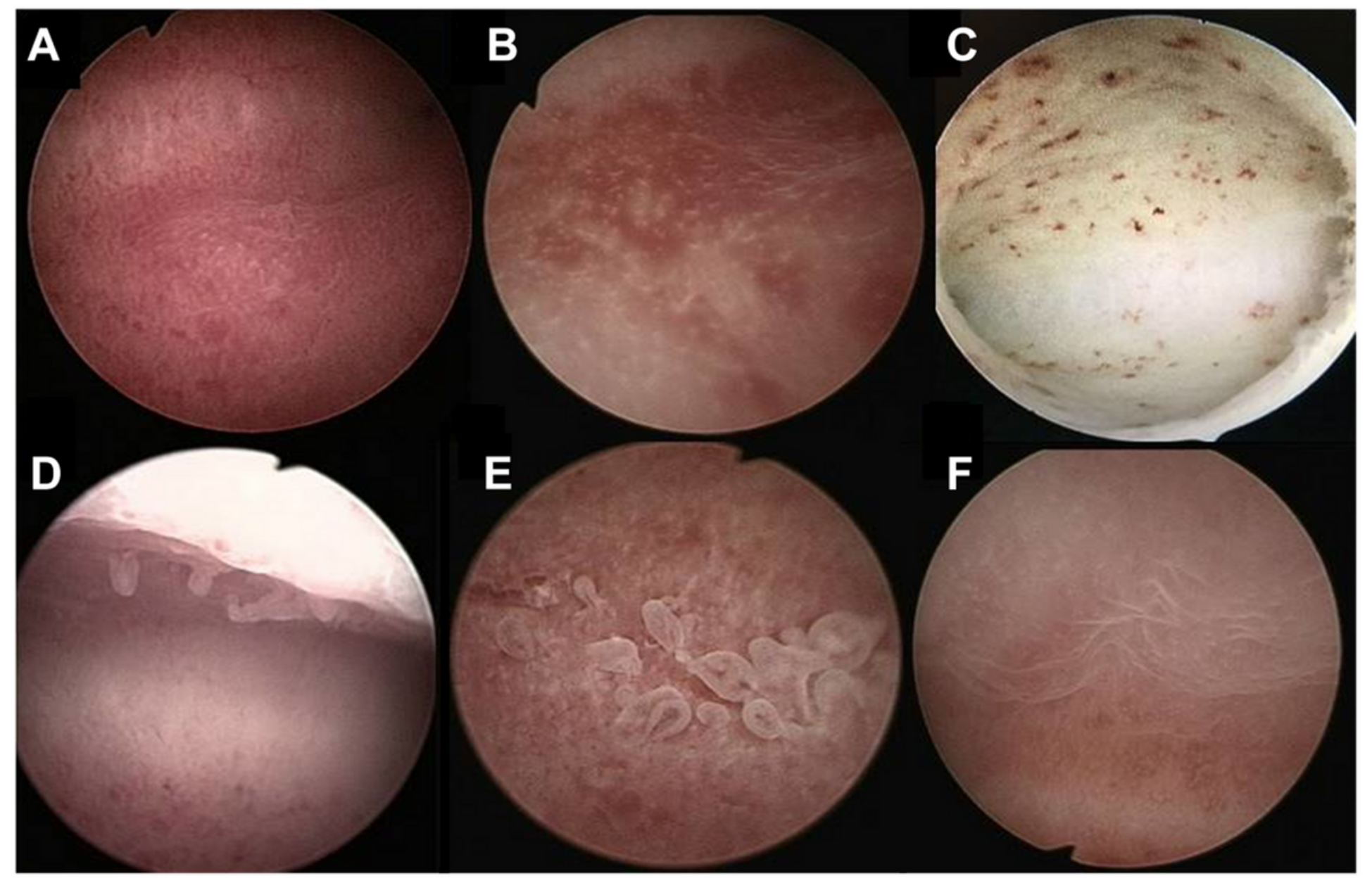

\section{Figure 1}

Characteristics of the different types of chronic endometritis (CE) under hysteroscopy. (A) Diffuse hyperemia: large areas of hyperemia with white spots. (B) Focal hyperemia: small areas of hyperemia. (C) Hemorrhagic spots: focal red areas with sharp and irregular borders, possibly in continuity with a capillary. (D-E) Micropolyps: endometrial polyps $<1 \mathrm{~mm}$ in diameter with prominent vascular pedicles, distributed focally (D), or diffusely (E). (F) The thick and pale appearance of the follicular endometrium defined as stromal edema (a normal finding during the secretory phase). 
Clinical pregnancy rate

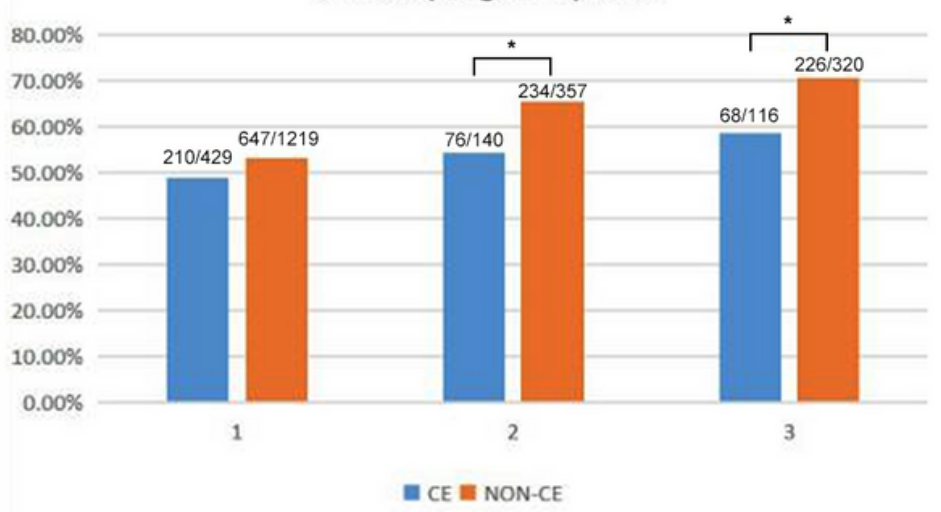

Miscarriage rate

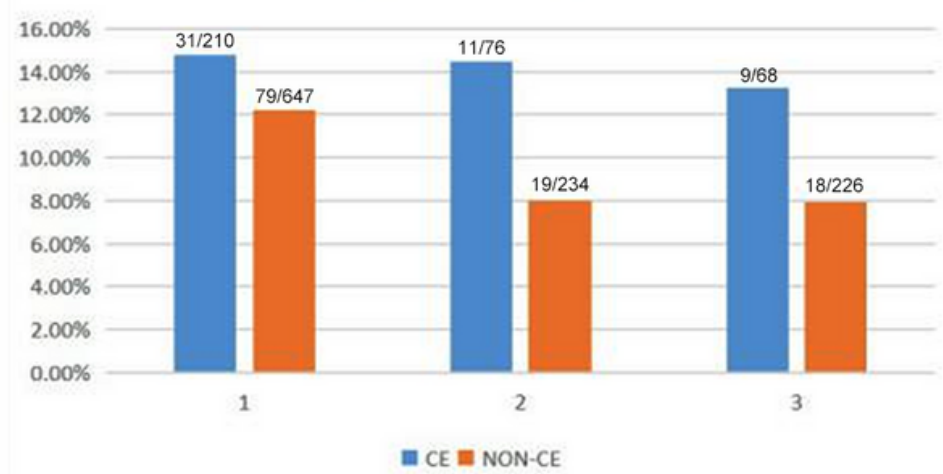

Live birth rate

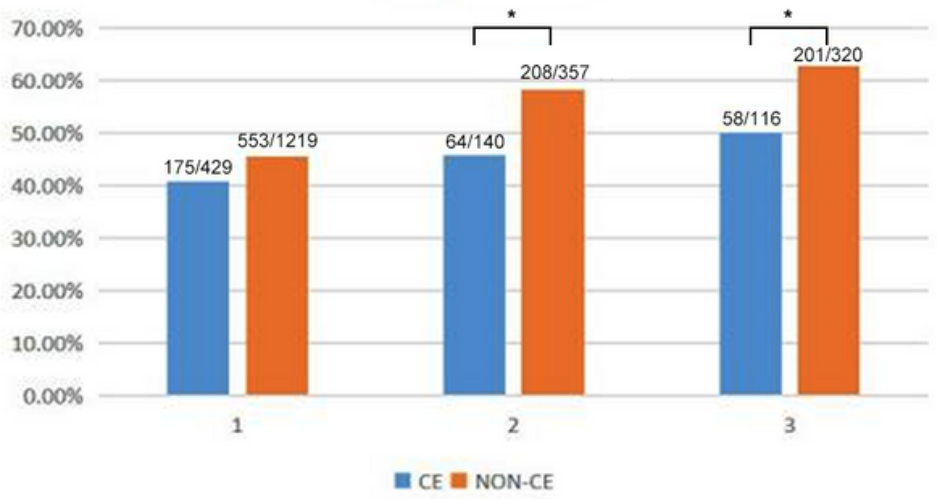

Premature birth rate

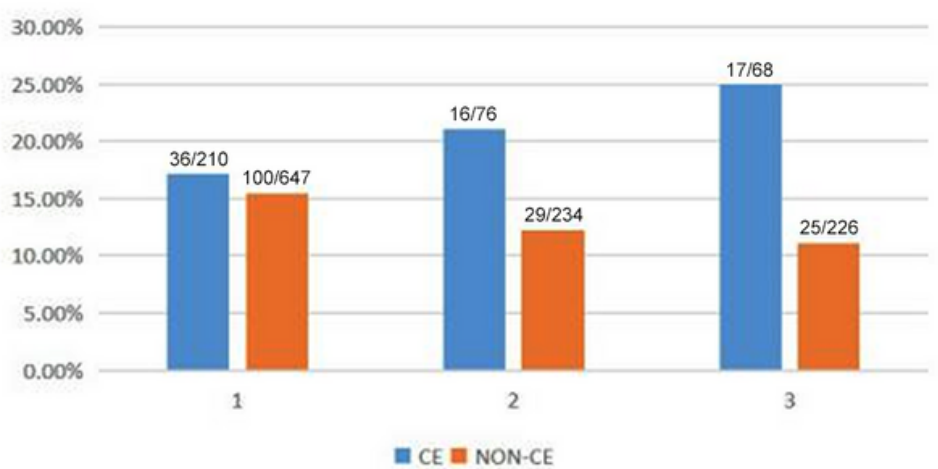

Figure 2

Comparison of the pregnancy outcomes for all embryo transfer cycles between patients with chronic endometritis (CE) and those without CE. 1 = fresh embryo transfer cycles; 2 = fresh embryo transfer cycles in standardized patients; 3 = high-quality embryo transfer cycles in standardized patients. ${ }^{\star} \mathrm{P}<0.05$.

\section{Supplementary Files}

This is a list of supplementary files associated with this preprint. Click to download.

- SupplementalFigure1.jpg

- SupplementalFigure2.jpg

- SupplementalTables.docx 